\title{
Biotopos marinos intermareales entre Canal Trinidad y Canal Smyth, Sur de Chile
}

\author{
Intertidal marine biotopes between Trinidad Channel and Smyth Channel, Southern Chile
Eulogio H. Soto ${ }^{\text {, Pedro Báez }}{ }^{2,3}$, María E. Ramírez², Sergio Letelier², Javier Naretto ${ }^{1,4}$ y Andrea Rebolledo ${ }^{2}$

\begin{abstract}
${ }^{1}$ Facultad de Ciencias del Mar y de Recursos Naturales, Universidad de Valparaíso, Casilla 5080, Reñaca, Viña del Mar, Chile. eulogio.soto@uv.cl

${ }^{2}$ Areas de Botánica e Invertebrados, Museo Nacional de Historia Natural, Casilla 787, Santiago, Chile

${ }^{3}$ Biología Marina, Facultad de Ecología y Recursos Naturales, Universidad Andrés Bello, Avda. República 440, Santiago, Chile ${ }^{4}$ Programa Master in Marine Biodiversity and Conservation, Scripps Institution of Oceanography, University of California, San Diego, Estados Unidos
\end{abstract}

\begin{abstract}
Through an oceanographic cruise carried out between the southern area of Aysén and the

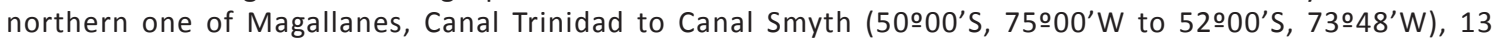
collecting stations were made to gather biological material to record and characterizing the intertidal biotopes of this extended area. A total of 19 biotopes were identified, from which 12 of those had been recorded previously at more septentrional areas of Aysén; other 6 represented variations of those previously recorded in other areas and only 1 was described as a new intertidal biotope. Among the former ones are rocky shore biotopes belonging to the dominant species Bostrychia harveyi, Acrosiphonia pacifica, Porphyra sp., Adenocystis utricularis, Iridaea tuberculosa and Mazzaella laminarioides-Nothogenia fastigiata. Among the 6 ones which represent variations of some biotopes before described there are 2 of Porphyra sp., 3 of Bostrychia harveyi and 1 of Mazzaella laminarioides-Nothogenia fastigiata. Just the rocky shore biotope integrated by the macroalgae Caepidium antarcticum and crustose coralline algae was the only new one. This new biotope is distributed in the lower eulitoral subzone of sheltered areas and it is characterized by having a strong subantarctic influence. Coastal biotope biodiversity was high, as a consequence of the multiple geographic, geologic, oceanographic and climatic factors interacting in this extended region. In general, the area was characterized by exhibiting a high biological diversity reflected through the diversity of observed biotopes, which demonstrated the presence of highly productive ecosystem.
\end{abstract}

Key words: Macroalgae, eulittoral, Patagonia, fjords

Resumen.- Durante un crucero oceanográfico realizado entre el sector sur de la región de Aysén y sector norte de Magallanes, Canal Trinidad a Canal Smyth (5000'S,7500'W a 5200'S, 7348'W), sur de Chile, se efectuaron 13 estaciones de recolección de material biológico para registrar y caracterizar los biotopos del intermareal rocoso del área. Se identificaron 19 biotopos, 12 de los cuales eran biotopos reconocidos para otras áreas más septentrionales de Aysén; 6 correspondían a variantes de los anteriores y uno sólo fue reconocido y descrito como biotopo nuevo. Entre los primeros están los biotopos de litoral rocoso correspondientes a las especies dominantes Bostrychia harveyi, Acrosiphonia pacifica, Porphyra sp., Adenocystis utricularis, Iridaea tuberculosa y Mazzaella laminarioides-Nothogenia fastigiata. Entre los 6 que representaban variantes de algunos de los biotopos anteriormente descritos hubo 2 de Porphyra sp., 3 de Bostrychia harveyi y 1 de Mazzaella laminarioides-Nothogenia fastigiata. Sólo el biotopo de litoral rocoso integrado por la macroalga Caepidium antarcticum y algas coralináceas crustosas, constituyeron un biotopo nuevo para el área que se distribuye en la subzona eulitoral inferior de frentes protegidos y se caracteriza por presentar una fuerte influencia subantártica. La diversidad de biotopos costeros fue alta, como consecuencia de los múltiples factores geográficos, geológicos, oceanográficos y climáticos que interactúan en esta extensa región. En general, el área se caracterizó por presentar una alta diversidad biológica reflejada en la diversidad de biotopos encontrados, que indicaría la presencia de ecosistemas altamente productivos.

Palabras clave: Macroalgas, eulitoral, Patagonia, fiordos 


\section{INTRODUCCIÓN}

La región austral de Chile, desde la Isla Grande de Chiloé hasta el Cabo de Hornos, representa un tercio de Chile continental y se caracteriza por ser un territorio accidentado, conformado por archipiélagos, fiordos y canales (Palma \& Silva 2004). Estos accidentes geográficos aumentan considerablemente la longitud del área si se considera, por ejemplo, el perímetro que involucra cada isla (Araya 1999). Debido a estas características, el estudio de la biodiversidad acuática y de las comunidades en esta zona ha sido difícil y esporádico (Reid \& Osorio 2000, Camus 2001, Schrödl 2003, Försterra 2009), no obstante algunos esfuerzos importantes han permitido documentar la ecología bentónica de fondos blandos principalmente en la región de Magallanes (Arntz \& Ríos 1999. Arntz et al. 2005) así como también su flora y fauna marina de sustratos rocosos (Häussermann \& Försterra 2009, Ramírez 2010). Las características geológicas y geográficas (Hervé et al. 2009, Soto 2009), entre ellas el sustrato para las especies acuáticas, la alta pluviosidad (Strub et al. 1998), unida a la gran influencia que ejerce en el sector la unión de los océanos Pacífico y Atlántico (Antezana 1999, Sobarzo 2009) y los Campos de Hielo Norte y Sur (Dávila et al. 2002, Landaeta et al. 2011), son factores que configuran un escenario ambiental muy distinto de aquel observado en el resto de Chile continental (Silva \& Palma 2006). Esto es determinante en la composición de su biota.

En lo particular, esta configuración geográfica tan heterogénea da origen a una amplia variedad de microclimas que varían gradualmente en sus características, desde ambientes límnicos propiamente dichos, típicos del sector oriental y aquéllos plenamente marinos del Pacífico, propios del sector occidental exterior (Soto 2009). Esta configuración, a través de la evolución geológica, constituye el sustrato para el desarrollo de ecosistemas estuarinos con características únicas en el mundo (Silva et al. 1997). Desde que la investigación científica de la región comenzó a fundamentarse en el estudio de las comunidades que integran los ecosistemas costeros (Sköttsberg 1941), es necesario adquirir un conocimiento integrado de esta área, principalmente por el uso creciente de la costa y su mar adyacente con fines industriales, relacionados con la salmonicultura, actividades pesqueras, planes turísticos y proyectos energéticos vinculados con el aprovechamiento de los recursos hídricos de la región, fomentando estudios globales e integrales sobre la biodiversidad del ambiente bentónico costero, quizás uno de los más afectados por la intervención antrópica de los últimos años (Buschmann et al. 2006).

La producción científica en la región de fiordos y canales lograda a través de los cruceros oceanográficos organizados por la Armada de Chile, a partir de 1995 (Silva \& Palma 2006) principalmente se refieren al estudio de las características de las comunidades de la columna de agua y del bentos sublitoral profundo (Rozbaczylo et al. 2007¹, Bustos et al. 2008, Marín \& Delgado 2009, Thatje \& Brown 2009, Ramajo \& Osorio 2010, Hinojosa et al. 2010, Landaeta et al. 2011). Con posterioridad a los estudios de Sköttsberg (1941) son muy pocas las investigaciones que han estado orientadas a estudiar la estructura de las comunidades intermareales de la región de Magallanes (Guzmán \& Ríos 1986, Ríos \& Guzmán 1982, Soto et al. 2010²). Iniciativas científicas internacionales desarrolladas entre los años 1998 a 2004, fueron orientadas al estudio de las comunidades marinas costeras en el sector Sur de la Región de Los Lagos (Chiloé), sector norte de la Región de Aysén (Parque Nacional Laguna San Rafael y Reserva Nacional Las Guaitecas) y sector sur en la Reserva Nacional Katalalixar (Báez \& Letelier 2001³, Osorio et al. 2002, John et al. 2002, 2003, Soto \& Paterson 2010). Estos estudios se continuaron con las prospecciones costero-intermareales realizadas en el período 2003-2006 en el marco del proyecto Biotopos Marinos Magallánicos (Letelier et al. 20064).

Acorde a lo señalado, el objetivo del presente trabajo fue describir los biotopos marinos intermareales encontrados en una zona de fiordos y canales del sur de Chile, caracterizando ecológicamente el área según la biodiversidad marina costera existente, para su consideración en la toma de decisiones respecto al manejo y conservación de los hábitats marinos costeros de este amplio sector del país.

\footnotetext{
${ }^{1}$ Rozbaczylo N, R Moreno, C Canales \& C Hernández. 2007. Diversidad específica y caracterización genética de poliquetos bentónicos de fondos blandos recolectados durante el Crucero CIMAR 12 Fiordos entre el Estuario Reloncaví y el Golfo de Corcovado. Informes preliminares Crucero CIMAR 12 Fiordos. 93-112

${ }^{2}$ Soto E, P Báez, M Ramírez, S Letelier, J Naretto \& A Rebolledo. 2010. Biotopos marinos intermareales y de aguas someras entre Canal Trinidad y Canal Smyth, XII Región (CONA C15F 09-13). CONA, Crucero CIMAR 15 Fiordos, Informes Preliminares 2010: 81-91

${ }^{3}$ Báez P \& S Letelier. 2001. Biotopos intermareales de la Región Austral Chile. Informes. Fondo de Apoyo a la Investigación Patrimonial, Centro de Investigaciones Diego Barros Arana, Santiago, pp. 34-39

${ }^{4}$ Letelier S, P Báez, C Andrade \& C Arriaza. 2006. Biotopos costeros de la Región Magallánica de Chile. Informes. Fondo de Apoyo a la Investigación Patrimonial. Ciencias Naturales, DIBAM. Centro de Investigaciones Diego Barros Arana, Santiago, pp. 35-37
} 


\section{MATERIALES Y MÉTODOS}

\section{ÁrEA DE ESTUDIO}

El presente estudio fue realizado como parte del Crucero CIMAR (Cruceros de Investigación Marina en Áreas Remotas) Fiordos 15, efectuado durante octubre y noviembre de 2009, entre Canal Trinidad situado en el sector sur de la región de Aysén y Canal Smyth, en el sector norte de la región de Magallanes, zona austral de Chile ( $50^{\circ} 00^{\prime} \mathrm{S} ; 75^{\circ} 00^{\prime} \mathrm{W}-52^{\circ} 00^{\prime} \mathrm{S} ; 73^{\circ} 48^{\prime} \mathrm{W}$ ), investigándose 13 estaciones para el estudio y evaluación de las comunidades intermareales (Fig. 1, Tabla 1). Las estaciones de muestreo fueron seleccionados del total de estaciones llevadas a cabo en el Crucero, considerarando aquellas en que la topografía permitiera estudiar sitios representativos, con acercamiento a la estación costera en períodos de marea baja y condiciones óptimas de luz natural.

\section{DESCRIPCIÓN Y CARACTERIZACIÓN DE BIOTOPOS}

El reconocimiento y acercamiento a los sitios costeros de estudio como playas, bahías, ensenadas y esteros de islas ubicadas en fiordos, golfos y canales australes, se realizó con embarcaciones neumáticas Zodiac ${ }^{\circledR}$ y con la colaboración de personal del B/O AGOR Vidal Gormaz de la Armada de Chile. Para prospectar cada una de las estaciones se realizó una descripción y evaluación de los biotopos reconocibles mediante la observación, utilizando el método descrito por John et al. $(1998,2002)$. Se georreferenció cada lugar de muestreo mediante un GPS digital GARMIN ${ }^{\circledR}$ III Plus y se obtuvieron datos de temperatura (equipo STD/CTDmodelo SD204) y salinidad $\left(\mathrm{S}_{0 / 00}\right.$; refractómetro manual ATAGO ${ }^{\circledR} \mathrm{S} /$ Mill-E) (Tabla 1). Además, se obtuvo un registro fotográfico de cada sector y en algunos casos la filmación en video de aquellos biotopos y organismos más conspicuos por medio de una cámara digital SONY ${ }^{\circledR}$ Cyber-shot DSC-S600.

Figura 1. Estaciones de muestreo para el estudio de Biotopos Marinos Intermareales. Crucero CIMAR Fiordos-15 (octubrenoviembre 2009) / Sampling stations for Intertidal Marine Biotopes study. CIMAR Fjords 15 Cruise (October-November 2009)

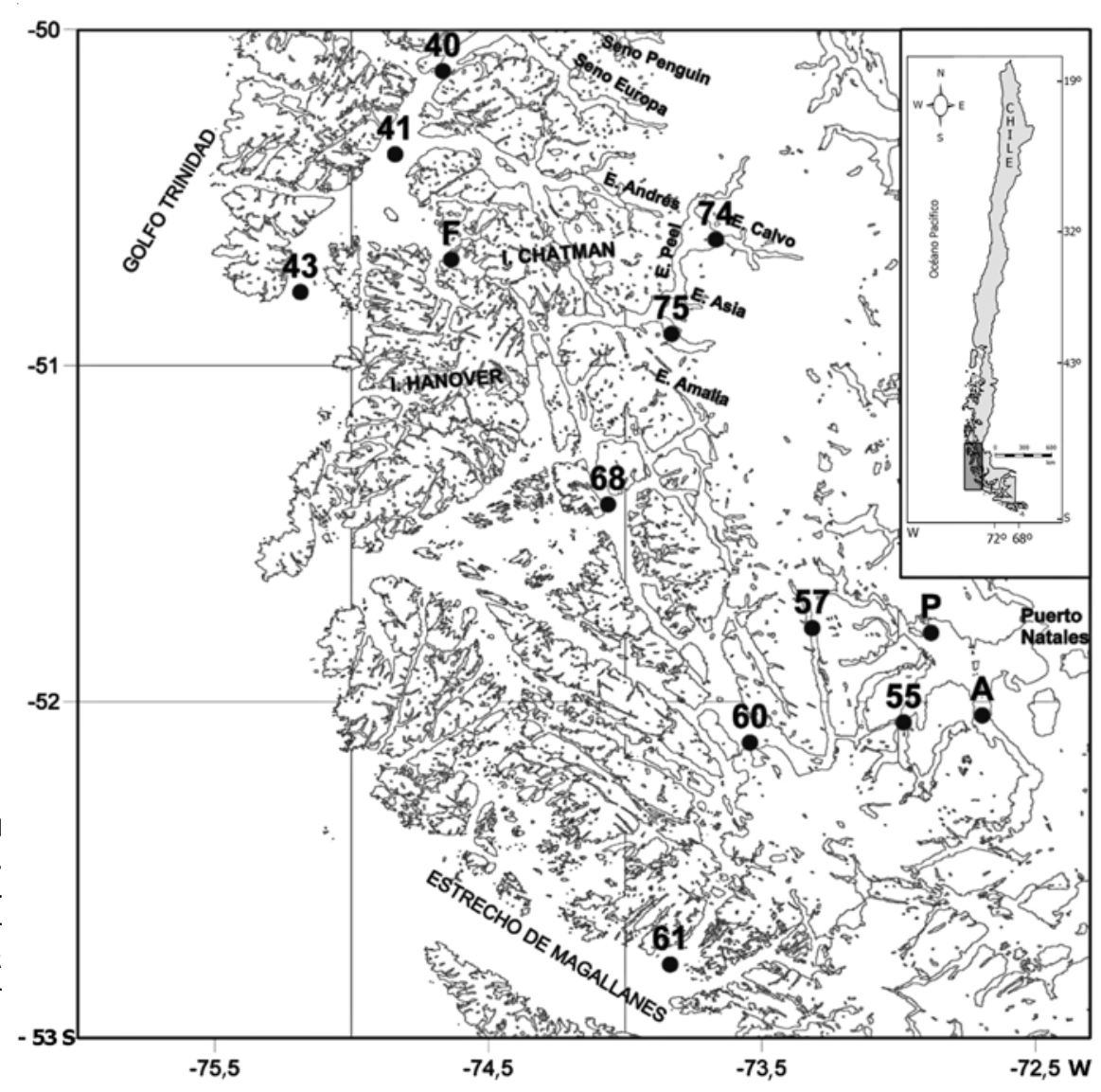


Tabla 1. Información ambiental de las estaciones donde se recolectaron muestras bentónicas de los biotopos observados / Environmental information of stations where benthic samples were collected from observed biotopes

\begin{tabular}{|c|c|c|c|c|c|}
\hline Fecha & Estación & Localidad & Latitud (S) & Longitud $(\mathrm{O})$ & Observaciones \\
\hline 19 oct & 41 & Puerto Mollineaux, Canal Concepción & $50^{\circ} 16^{\prime} 37^{\prime \prime}$ & $74^{\circ} 53^{\prime} 21^{\prime \prime}$ & $\begin{array}{l}\text { Ensenada protegida con pendiente } \\
\text { suave y aportes de agua dulce }\end{array}$ \\
\hline 20 oct & 75 & Canal Amalia, Ventisquero Skúa & $50^{\circ} 56^{\prime} 06^{\prime}$, & $73^{\circ} 44^{\prime} 24^{\prime \prime}$ & Pared rocosa. Influencia glaciar \\
\hline 21 oct & 74 & Estero Calvo, Ventisquero Matthew & $50^{\circ} 38^{\prime} 20^{\prime \prime}$ & $73^{\circ} 38^{\prime} 28^{\prime \prime}$ & $\begin{array}{l}\text { Sustratos de arena, lodo y piedras. } \\
\text { Influencia glaciar }\end{array}$ \\
\hline 24 oct & 40 & Canal Concepción, Frente Isla Topar & $50^{\circ} 08^{\prime} 28^{\prime \prime}$ & $74^{\circ} 39^{\prime} 06^{\prime \prime}$ & $\begin{array}{l}\text { Frente protegido a semiexpuesto. } \\
\text { Paredes abruptas de roca }\end{array}$ \\
\hline 25 oct & 43 & Isla Duque de York (Canal Concepción) & $50^{\circ} 47^{\prime} 33^{\prime \prime}$ & $75^{\circ} 16^{\prime} 49^{\prime \prime}$ & $\begin{array}{l}\text { Sector rocoso en extensa playa de } \\
\text { arena. Frente semiexpuesto; ambiente } \\
\text { prístino, influencia marina }\end{array}$ \\
\hline 26 oct & 60 & Puerto Fontaine (Península Las Montañas) & $52^{\circ} 03{ }^{\prime} 49^{\prime \prime}$ & $73^{\circ} 28^{\prime} 00^{\prime \prime}$ & $\begin{array}{l}\text { Sector semiprotegido, rocoso y } \\
\text { pedregoso }\end{array}$ \\
\hline 27 oct & 55 & Canal Kirke & $52^{\circ} 03 ’ 33^{\prime}$, & $72^{\circ} 57^{\prime} 37^{\prime \prime}$ & $\begin{array}{l}\text { Paredes inclinadas de roca. Aportes de } \\
\text { agua dulce. Frente semiprotegido }\end{array}$ \\
\hline 3 nov & $\mathrm{P}$ & $\begin{array}{l}\text { Punta Vergara, Península Antonio Varas } \\
\text { (Golfo Almirante Montt) }\end{array}$ & $51^{\circ} 45^{\prime} 32^{\prime \prime}$ & $72^{\circ} 51^{\prime} 00^{\prime \prime}$ & $\begin{array}{l}\text { Playa de pendiente moderada con } \\
\text { piedras y cantos rodados. Frente } \\
\text { semiexpuesto }\end{array}$ \\
\hline 4 nov & A & Islote Valdivia, Estero Obstrucción & $51^{\circ} 59^{\prime} 15^{\prime \prime}$ & $72^{\circ} 41^{\prime} 41^{\prime \prime}$ & Playa semiexpuesta de piedras y roca \\
\hline 6 nov & 57 & Estero Las Montañas & $51^{\circ} 48^{\prime} 23^{\prime \prime}$ & $73^{\circ} 19^{\prime} 16^{\prime \prime}$ & $\begin{array}{l}\text { Sector estuarino semiprotegido con } \\
\text { paredes verticales y piedras }\end{array}$ \\
\hline 8 nov & 61 & $\begin{array}{l}\text { Isla Manuel Rodriguez, Canal Smyth } \\
\text { (Faro Fairway) }\end{array}$ & $52^{\circ} 42^{\prime} 57^{\prime \prime}$ & $73^{\circ} 47^{\prime} 22^{\prime \prime}$ & $\begin{array}{l}\text { Sector expuesto de rocas y piedras con } \\
\text { alta diversidad faunística. Influencia } \\
\text { marina }\end{array}$ \\
\hline \multirow[t]{2}{*}{9 nov } & 68 & Isla Vancouver, Canal Sarmiento & $51^{\circ} 26 ’ 21^{\prime \prime}$ & $74^{\circ} 04^{\prime} 42^{\prime \prime}$ & $\begin{array}{l}\text { Costado de canal. Sector semiprotegido } \\
\text { con alta diversidad algológica }\end{array}$ \\
\hline & $\mathrm{F}$ & $\begin{array}{l}\text { Caleta Paroquet, Isla Chatham (Canal } \\
\text { Inocentes) }\end{array}$ & $50^{\circ} 41^{\prime} 08^{\prime \prime}$ & $74^{\circ} 33^{\prime} 28^{\prime \prime}$ & $\begin{array}{l}\text { Ambiente mixto de terrazas, grietas y } \\
\text { playa de cantos rodados. Sector } \\
\text { semiprotegido }\end{array}$ \\
\hline
\end{tabular}

En terreno se individualizaron los ambientes, apartando aquellos conjuntos de especies que representaban mosaicos de biotopos, por cuanto éstos últimos, además de ser difíciles de interpretar, a menudo son el reflejo de la heterogeneidad del sustrato. Por lo tanto, cada sector fue caracterizado considerando la geografía del lugar (bahía, playa, condiciones de estuario), presencia de agua dulce, grado de exposición a los vientos predominantes y oleaje, determinación del tipo de frente: expuesto, semi-expuesto y protegido; pendiente, amplitud y longitud total de la playa; características generales y geomorfológicas del sedimento y del sustrato, alteraciones antrópicas observables, marco escénico y paisaje general (Tabla 1).

Para la definición de las características ecológicas de los biotopos observados en cada sector se aplicaron los criterios de Stephenson \& Stephenson (1949, 1972), modificados por Lewis (1964), metodología que se utiliza de forma regular en los estudios de distribución y ecología intermareal. Según este método cualquier playa puede dividirse en un cierto número de franjas horizontales, caracterizadas por ciertas especies de algas e invertebrados, o grupos de éstos que son típicos de cada uno de estos niveles (Letelier et al. 2006). En los biotopos de playas rocosas se ha observado que las franjas pueden variar latitudinalmente. Se hace necesario entonces caracterizar estos biotopos por el tipo de asociaciones de algas/invertebrados presentes. En otras palabras, el biotopo representa 'la biota interactuante con el hábitat físico, es decir, organismos de origen vegetal y animal que viven en una localidad, considerados en conjunto con su entorno físico inmediato' (John et al. 2003). Sin embargo el método empleado en el presente estudio difiere en parte del propuesto por Olenin \& Ducrotoy (2006), ya que no se considera coberturas de organismos. 
Como parte de la caracterización y evaluación de cada biotopo se recolectaron muestras de invertebrados y algas en cada sitio realizándose una posterior confirmación de su determinación taxonómica a bordo o en los laboratorios de Ficología, Malacología y Carcinología del Museo de Historia Natural de Santiago con la ayuda del Manual de Biotopos (John et al. 2003). Las muestras recolectadas fueron fijadas en formalina diluida en agua de mar (5\% y $10 \%$ ) almacenadas en bolsas plásticas y frascos plásticos de diferentes tamaños y conservadas en alcohol de $70^{\circ} \mathrm{y}$ silica gel. Una vez en el laboratorio se realizó una separación por grandes grupos (algas, moluscos, crustáceos, equinodermos, anélidos, poríferos, cnidarios y otros). Los invertebrados fueron contados, fotografiados, etiquetados y guardados. En el caso de las algas, algunas fueron montadas en hojas de herbario y otras almacenadas y preservadas en formalina para su identificación final en el Laboratorio de Ficología del Museo Nacional de Historia Natural de Santiago. Los resultados obtenidos de la caracterización biológica y ambiental de cada estación fueron llevados a fichas estándar, utilizadas previamente en el terreno (John et al. 2003), obteniendo una base de datos para cada localidad del área de estudio.

\section{Código de BIOTOPOS (Nomenclatura)}

Las descripciones siguen el formato recomendado por Connors et al. (1997), que ha sido anteriormente usado en la región de Aysén (John et al. 2003) y en el BioMar Biotope Viewer $2.0^{5}$

A los biotopos se les asignó un código de letras único basado en las características del biotopo una combinación de especies clave, formas de vida y hábitat físico característicos. Los títulos son descriptivos e incluyen las características biológicas claves del biotopo junto con características del ambiente físico. Los biotopos se basaron, hasta donde fue posible, en los taxones dominantes y más característicos. Los nombres de las especies son modificados usando las 3 primeras letras de un género o nombre del taxa. Si se utiliza una especie el código se deriva usando la primera letra del género y las tres primeras letras del nombre específico. Cuando la composición biológica fue muy compleja para derivar un código simple, entonces se utilizaron las características del hábitat. Dentro del código de biotopo cada nuevo elemento comienza con una letra mayúscula. La nomenclatura de los códigos se ordena de esta forma:

\footnotetext{
${ }^{5}<$ http://www.ecoserve.ie/biomar/viewer.html>
}

Posición de la playa: Zona litoral (L), zona sublitoral (SL), zona supralitoral (SUP).

Hábitat: Marismas (SM), pileta rocosa (RP).

Exposición al oleaje: Protegida (SH), moderadamente expuesta (ME), severamente expuesta (SE), protegida a severamente expuesta (SS).

Sustratos: Roca (R), arena (SND), sedimento (SED), barro o fango (MUD), mezcla (MXD).

Formas de vida: Conjunto de algas (ATRF), película de algas (AFILM), alga feófita de gran tamaño (KP).

Grupo y/o grupos funcionales: Macroalgas pardas (B), cirrípedos balánidos (BARN), bivalvos (BIV), crustáceos (CRUS), cianobacterias (CYAN), gasterópodos (GAS), macroalgas verdes (G), líquenes (LICH), macroalgas rojas carnosas (R).

Géneros/Especies: Adenocystis utricularis (Autr), Austromegabalanus psittacus (Apsi), Balánidos (Barn), Bostrychia harveyi (Bhar), Acrosiphonia pacifica (Apac), Cladophoropsis brachyarcta (Cbra), Corallina officinalis var. chilensis (Coff), Iridaea tuberculosa (Itub), Durvillaea antarctica (Dant), Ectocarpus siliculosus (Esil), Elminius kingii (Ekin), Nothogenia fastigiata (Nfast), Caepidium antarcticum (Canta), Lessonia nigrescens (Lnig), Macrocystis pyrifera (Mpyr), Mazzaella laminarioides (Mlam), Mytilus chilensis (Medu), Porphyra sp. (Porp), Scytothamnus fasiculatus (Sfasi).

\section{Resultados}

Las 13 estaciones del Crucero CIMAR Fiordos 15 donde se tomaron las muestras representan en total 19 biotopos incluyendo sus variantes. Algunos de los biotopos se registran más de una vez y otros se encuentran divididos, debido a que la extensión de la estación era muy grande o porque el área, siendo pequeña, representaba un conjunto de ambientes muy variados. Estos biotopos, al igual que las estaciones, están ubicados principalmente hacia el sector oriental del área y al interior de los canales. Representan ambientes protegidos o semi-expuestos en su mayoría, con un gran aporte hídrico derivado de los deshielos, de los ambientes límnicos de ríos, esteros y otros cursos de agua y de las precipitaciones. Manifiestan, en consecuencia, condiciones propias de ambientes más estuarinos. Se exceptúan de esta descripción general la zona costera de la Isla Duque de York (estación 43) y de Isla Manuel Rodríguez (estación 61 ), las cuales presentan características de ambiente 
marino y se ubican en sectores más expuestos (Tabla 1). En una categoría intermedia y presentando condiciones medianamente expuestas se registran el Canal Concepción, frente a Isla Topar (estación 40) y Puerto Mollineaux (estación 41). También se observó que todos los ambientes rocosos se caracterizaban por una gran riqueza de algas e invertebrados sésiles. Toda la extensa área costera estudiada presenta un estado general relativamente prístino.

Cada biotopo fue identificado de acuerdo con las poblaciones de macroalgas predominantes en el sitio de observación, en asociación con aquellos invertebrados más conspicuos (principalmente moluscos gasterópodos y cirrípedos balánidos), caracterizando los distintos niveles de la zona intermareal estudiada. La riqueza de algas de cada estación y de cada biotopo, es alta si se le compara con la de sectores ubicados en la región de Aysén (John et al. 2003), como también lo es el conjunto de invertebrados que caracteriza cada biotopo. Se describen a continuación los biotopos más característicos y sus variantes registradas.

\section{DESCRIPCIÓN DE BIOTOPOS}

\section{Biotopo de AdENocystis utricularis (BoRy de SAinT- VinCENT) SKÖTTSBERG, 1907 (LR AUtr)}

Zona litoral/rango: Zona eulitoral media e inferior.

Clasificación del hábitat: Agua de mar con baja salinidad (31).

Exposición al oleaje: Frente protegido a semi-expuesto.

Otros modificadores: Exposición al oleaje, inclinación o pendiente de la pared y pastoreo de erizos hacia el submareal somero.

Distribución geográfica: Extendida ampliamente en ambientes costeros ubicados desde la zona norte de la región de Aysén hasta Magallanes.

Descripción: Agregaciones de la macroalga parda Adenocystis utricularis, en paredes, macizos rocosos y bloques rocosos muy estables. A menudo acompañada del alga roja Porphyra sp. y del alga parda Scytothamnus fasciculatus (Hooker \& Harvey) Cotton, 1915, junto a la franja de Mytilus chilensis Hupé in Gay, 1854 que son más conspicuos hacia el frente semi-expuesto y disminuyen paulatinamente hacia el frente protegido. Presencia menor de las macroalgas Leptofauchea chiloensis Dalen \& Saunders, 2007, Lophurella hookeriana (J. Agardh) Falkenberg, 1901, Ulva sp.,
Scytosiphon lomentaria (Lyngbye) Agardh, 1848, Polysiphonia sp. y Halopteris obovata (Hooker\& Harvey) Sauvageau, 1904 y de los invertebrados Balanus laevis Brugiere, 1789, Jehlius cirratus (Darwin, 1854), Nacella magellanica (Gmelin, 1791) y Fissurella picta (Gmelin, 1791).

Este biotopo corresponde a la estación ubicada en el Canal Concepción frente a Isla Topar, muy cercano a Bahía Open (estación 40, Fig. 2d).

\section{Biotopo De Bostrychia HARVEYI (MONTAGNE, 1852) (LR BHAR)}

Zona litoral/rango: Zona eulitoral superior.

Clasificación del hábitat: Agua de mar con baja salinidad $(<30)$.

Exposición al oleaje: Frente protegido a semi-expuesto.

Otros modificadores: En sector A: pared rocosa casi vertical; sector B: playa de bolones poco estables en el borde de la playa, de arena de grano mediano; la salinidad en este biotopo es de un amplio rango, con salinidades desde 10 hasta condiciones totalmente marinas (>32). Además, como modificadores de un esquema de biotopo general, se encontraron lugares sombríos, filtraciones y pequeños arroyos de agua dulce.

Distribución geográfica: Biotopo extendido ampliamente en ambientes costeros ubicados desde la parte norte de la región de Aysén hasta Magallanes.

Descripción: Agregaciones de color amarillento a café del alga Bostrychia harveyi en sectores de pared rocosa ligeramente inclinada, aledaños a la playa, que incluye bolones, algunos poco estables y otros más estabilizados, cercanos a raíces y troncos de árboles. La banda de Bostrychia harveyi es más conspicua y definida en el sector de la pared rocosa y se va atenuando hacia los sectores de bloques y se hace más inconspicua hacia el sector de bolones y hacia los lugares más sombríos. Se desarrolla en una amplia gama de exposición al oleaje, salinidad y grados de luz y sombra. Generalmente acompañada por plantas con forma de cojines de Wittrockiella lyalii (ex Cladophoropsis brachyartra) y entre agregaciones más difusas de Hildenbrandtia lecannellieri, Nothogenia fastigiata (Bory de SaintVincent) Parkinson, 1983, Scytothamnus fasciculatus y Ulva sp. Las bandas de B. harveyi están distribuidas extensamente, siendo posible visualizarlas como una franja de color trigo maduro en los roqueríos de pendiente escarpada de los fiordos. Para el biotopo desarrollado en 
la Reserva Nacional Katalalixar (zona sur de Aysén) se había mencionado que: 'cuando el biotopo está sobre troncos y raíces de árboles, las algas filamentosas están acompañadas frecuentemente por filamentos capilares del alga verde Rhizoclonium ambigum. También otras dos algas verdes (Ulva sp. 1. de forma cilíndrica, ex Enteromorpha sp. y Ulva sp. 2 de forma laminar) están a menudo presentes en este nivel de la playa, especialmente donde hay muchos aportes de agua dulce y no hay sombra'. Este biotopo ha sido citado para todo el Parque Nacional Laguna San Rafael (PNLSR), el Archipiélago de Los Chonos y la región de Katalalixar (John et al. 2003)

Este biotopo corresponde a la estación ubicada en Puerto Mollineaux, Canal Concepción (estación 41, Fig. 2a).

Una variación de este biotopo es el que corresponde a la estación de Isla Vancouver, Canal Sarmiento (estación 68), donde se encuentra un ambiente más marino, con salinidad de 32 y que se caracterizó por la alta cobertura y diversidad de macroalgas. En este biotopo Bostrychia harveyi se presenta asociada con Ulva sp. en los niveles altos, con Adenocystis utricularis y Ceramium sp. en el nivel medio y con Sarcothalia crispata (Bory de SaintVincent) Leister, 1993 y Pylaiella littoralis (Linnaeus) Kjellman, 1872 en los niveles bajos (Fig. 2l). Además, se registran de manera importante los invertebrados Nacella magellanica y Jehlius cirratus y las macroalgas Iridaea tuberculosa (Hooker \& Harvey) Leister, 1993, Scytosiphon lomentaria, Leptofauchea chiloensis y Lophurella hookeriana en la zona eulitoral inferior.

Otra variante de este biotopo fue la observada en la estación del Islote Valdivia (Estero Obstrucción) (estación A), con salinidad de 18, en el cual Bostrychia harveyi se encuentra asociada a Porphyra sp. en el nivel alto, Ceramium sp. en el nivel medio y Acrosiphonia pacifica (Montagne) Agardh, 1857en la zona baja; B. harveyi se ubica sobre bloques y bolones grandes estabilizados hacia los lados de playas de cantos rodados, que limitan directamente con el ambiente terrestre, en el límite inferior de la zona supralitoral (Fig. 2h). A diferencia del biotopo anterior (estación 68), esta variante registró escasa cobertura y diversidad de algas.

Una variante parecida a la anterior se observó en Puerto Fontaine (Península Las Montañas) (estación 60), sector semi-expuesto. Se registró una salinidad de 23, condición en la cual Bostrychia harveyi, Porphyra sp. y Scytothamnus fasciculatus se encontraban mezcladas hacia los niveles altos y Ulva sp., Adenocystis utricularis,
Nothogenia fastigiata y Mazzaella sp. estaban dispuestas en los niveles medios (Fig. 2f). Estas algas se encontraron distribuidas sobre bloques y bolones grandes estabilizados en playas de arena de grano mediano y bolones más pequeños hacia los costados, junto a invertebrados como Mytilus chilensis, Elminius kingii Gray, 1831 y Nacella magellanica.

\section{Biotopo de PoRphyRa SP. (LR PORP)}

Zona litoral/rango: Zona eulitoral superior.

Clasificación del hábitat: Salinidad reducida (20) con amplio rango de variación, hasta totalmente marina ( $\geq 32)$.

Exposición al oleaje: Moderadamente expuesta.

Otros modificadores: Aunque es de amplio rango de exposición al oleaje, posiblemente la disminución de éste, que puede reducirse hasta alcanzar condiciones de ambiente muy protegido, constituye una de las principales modificaciones; también la calidad del sustrato rocoso, desde macizos y bloques rocosos, bolones e incluso moluscos en la zona eulitoral hasta piedras semienterradas en el sedimento, el cual adquiere características de fango claro.

Distribución geográfica: Extendida ampliamente en ambientes costeros ubicados desde la provincia de Chiloé (Región de Los Lagos) hasta Magallanes.

Descripción: Las agregaciones de Porphyra sp. presentan aspecto membranoso color trigo maduro a pardo y rojizo, muy evidentes sobre rocas o moluscos bivalvos en la zona eulitoral superior siendo esta alga abundante en playas rocosas protegidas que tienen salinidad reducida y un amplio rango de exposición al oleaje. Este biotopo se encuentra desarrollado en la parte superior de la playa, donde las agregaciones de Porphyra sp. crecen adheridas a las rocas, tolerando incluso la desecación. Debido a esta variabilidad en la zonación este biotopo ocupa varios niveles en la playa, asociado frecuentemente a cirrípedos balánidos y mitílidos, o bien inmediatamente sobre la zona de balánidos. Se asocia comúnmente con las algas Pylaiella littoralis que se extiende por las zonas alta y media, Acrosiphonia pacifica que se extiende más hacia la zona media y con Adenocystis utricularis en la zona baja. Ocasionalmente conforma una franja conspicua en la zona eulitoral inferior en condiciones de oleaje moderadamente expuestas, donde se visualiza parte del biotopo de Macrocystis pyrifera (Linnaeus) Agardh, 1820. En el presente trabajo este biotopo corresponde a la estación ubicada en el 

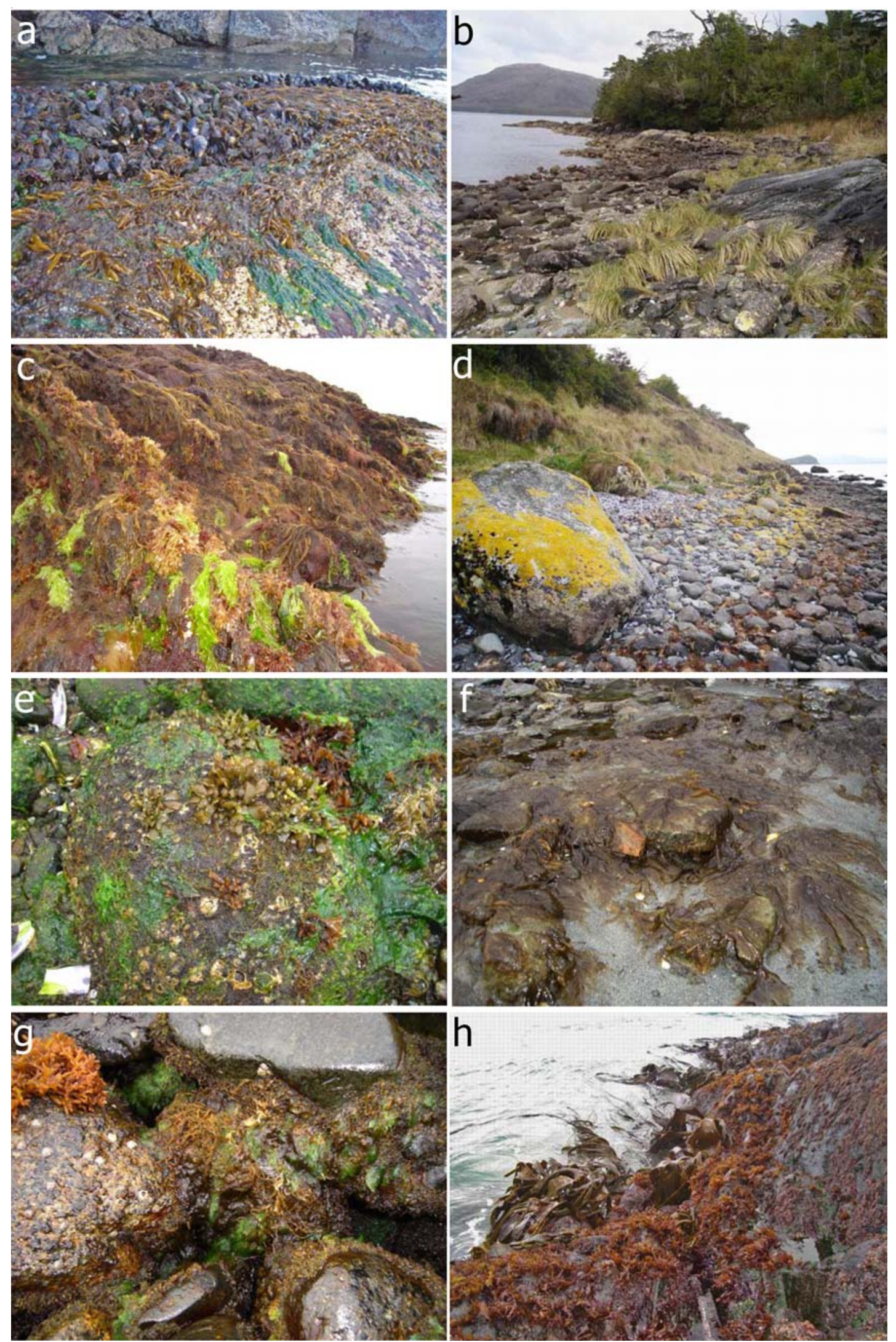

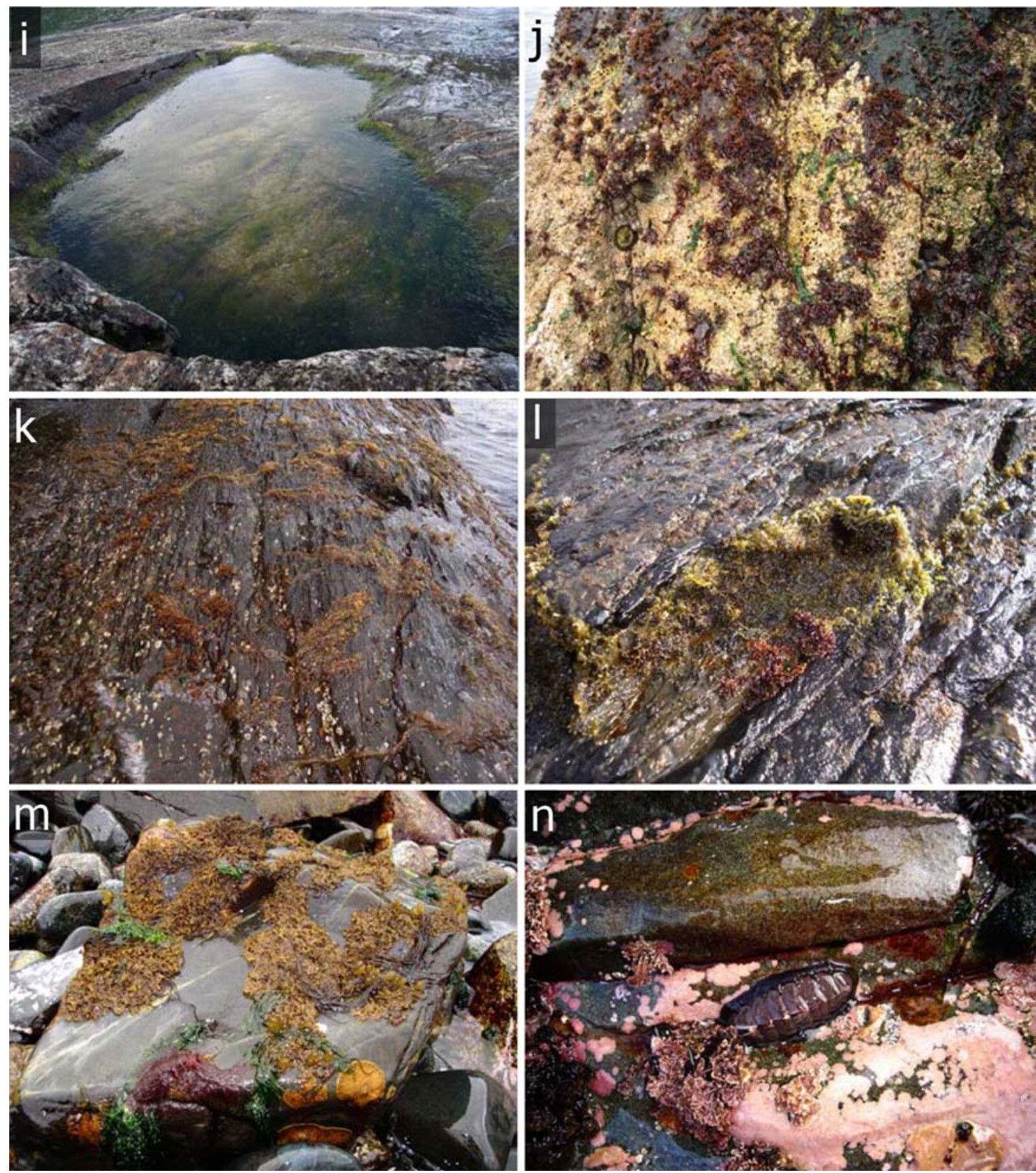

Figura 2. Código y estación de muestreo de Biotopos Marinos Intermareales registrados entre Canal Trinidad y Canal Smyth: a) LR Autr (Est. 40), b) LR Bhar (Est. 41), c) LR Bhar (Est. 68), d) LR Bhar (Est. A), e) LR Bhar (Est. 60), f) LR Porp (Est. 74), g) LR Porp (Est. F), h) LR Porp (Est. 43), i) LR Apac (Est. 75), j) LR MlamNfast (Est. 57), k) LR MlamNfast (Est. 55), I) LR Itub (Est. P), m) LR Canta (Est. 61) y n) LR Canta (Est. 61) / Code and sampling stations of Intertidal Marine Biotopes recorded between Trinidad Channel and Smyth Channel: a) LR Autr (Est. 40), b) LR Bhar (Est. 41), c) LR Bhar (Est. 68), d) LR Bhar (Est. A), e) LR Bhar (Est. 60), f) LR Porp (Est. 74), g) LR Porp (Est. F), h) LR Porp (Est. 43), i) LR Apac (Est. 75), j) LR MlamNfast (Est. 57), k) LR MlamNfast (Est. 55), I) LR Itub (Est. P), m) LR Canta (Est. 61) and n) LR Canta (Est. 61) 
Estero Calvo (Ventisquero Matthew) (estación 74, Fig. 2c).

Una variante de este biotopo fue registrada en Caleta Paroquet, Isla Chatham (Canal Inocentes) (estación F), donde Porphyra sp. se encuentra limitada a la franja alta junto a Catenella fusiformis (J.Agardh) Sköttsberg, 1923, asociada con Nothogenia fastigiata y Scytothamnus fasciculatus en la zona media, en sectores de terrazas y grietas y a Macrocystis pyrifera en la zona baja, en un sector semiprotegido, con salinidad de 17 (Fig. 2j). Los invertebrados registrados en este biotopo fueron Mytilus chilensis, Notobalanus flosculus (Darwin, 1854), Elminius kingii, Nacella clypeater (Lesson, 1831) y $N$. magellanica.

Una segunda variante de este biotopo se registró en la Isla Duque de York, Canal Concepción (estación 43), ya que las agregaciones de Porphyra sp. se encuentran distribuidas ampliamente en los sectores superiores, junto a Ulva sp., especie que además se sitúa en niveles medios junto a coberturas importantes de Nothogenia fastigiata y Mazzaella laminarioides (Bory de Saint-Vincent) Fredericq, 1993 y con poblaciones de Durvillaea antarctica (Chamisso) Hariot, 1892 y Codium fragile (Suringar) Hariot, 1889 en niveles inferiores. El ambiente se caracterizó por presentar macizos rocosos de pendiente inclinada, sometidos a oleaje moderado, en un frente semiexpuesto de condiciones más oceánicas con salinidad de 32. En niveles inferiores se suma a lo anterior, la presencia de algunos moluscos como Concholepas concholepas (Brugiere, 1789) y Chiton sp. (Fig. 2e).

En términos generales el biotopo de Porphyra sp. ha sido señalado también para el PNLSR, Archipiélago de Los Chonos y Katalalixar en la región de Aysén (John et al. 2003).

\section{BIOTOPO DE ACROSIPHONIA PACIFICA. (LR APAC)}

Zona litoral/rango: Zona eulitoral superior a sublitoral.

Clasificación del hábitat: Salinidad reducida (20), con amplio rango de variación hasta totalmente marina ( $\geq 32)$.

Exposición al oleaje: Desde protegida a moderadamente expuesta.

Otros modificadores: Tipo de sustrato y amplitud de la zona litoral, presencia de hielo y competencia por espacio (sustrato) con otras macroalgas e invertebrados.

Distribución geográfica: Biotopo ampliamente distribuido en ambientes costeros ubicados más al norte en la región de Aysén y en fiordos y canales interiores de la región de
Magallanes.

Descripción: Agregaciones verde oscuro de Acrosiphonia pacifica que se extienden desde la subzona eulitoral superior hasta la zona sublitoral, sobre playas y sectores rocosos en aguas salobres. Macizos rocosos estables en la zona eulitoral (más comunes en la playa media a superior), a menudo cubiertos por agregaciones verde oscuras de este alga verde filamentosa, donde la salinidad es de 20 o menor y la temperatura de $7,3^{\circ} \mathrm{C}$. A menudo se observa acompañada de Porphyra sp. en pozas medias junto a Scytothamnus y Adenocystis, macroalgas pardas características de la subzona eulitoral inferior. A. pacifica es más conspicua cuando la cubierta de la otra alga es baja, especialmente en la subzona eulitoral superior y en la zona sublitoral $(0-10 \mathrm{~m})$.

En general este biotopo se caracterizó por la escasa diversidad de invertebrados. Ha sido anteriormente documentado para las áreas del Golfo Elefantes y La Laguna San Rafael en la región de Aysén (John et al. 2003).

Este biotopo fue registrado para el Canal Amalia (Ventisquero Skúa) (estación 75), mientras que variantes de su composición no fueron observadas (Fig. 2b).

\section{Biotopo de MazzaELLa LAMiNARIoIDES y Nothogenia FASTIGIATA (LR MLAMNFAST)}

Zona litoral/rango: Zona eulitoral inferior.

Clasificación del hábitat: Salinidad reducida a moderada (25) con rango de variación hasta totalmente marina ( $\geq 32)$.

Exposición al oleaje: Moderadamente expuesta hasta muy protegido.

Otros modificadores: Además de la exposición al oleaje, el tipo de sustrato.

Distribución geográfica: Biotopo distribuido ampliamente en ambientes costeros ubicados desde la zona norte de la región de Aysén hasta la región de Magallanes.

Descripción: Mazzaella laminarioides y Nothogenia fastigiata forman parches de color pardo amarillento café claro en playas de macizos rocosos y playas de bolones de bahías protegidas o moderadamente expuestas y en bahías y ensenadas de la subzona eulitoral media a inferior. Mazzaella laminarioides y Nothogenia fastigiata se encuentran repartidas alternadamente (mezcladas) en el nivel medio y coexisten con Ulva sp. y Acrosiphonia pacifica en los niveles más bajos. Ambas están generalmente acompañadas por algunas algas feófitas 
que incluyen Scytothamnus fasciculatus, Adenocystis utricularis, Scytosiphon lomentaria, Halopteris sp. y otras algas rojas (e.g., Ahnfeltiopsis durvillaei, A. furcellatus, Ahnfeltia plicata). A veces esta última consiste en costras rojo-parduzcas sin las frondas erguidas. Es común observar que se asocian diversos moluscos con estas algas, e.g., Mytilus chilensis, Fissurella, Chiton, Tegula, Plaxiphora, Tonicia y Nacella, así como colonias de cirrípedos destacando las especies Elminius kingii y Jehlius cirratus. Hacia el límite con el submareal se encuentran en contacto con Durvillaea antarctica en los sectores más expuestos y con Macrocystis pyrifera en los sectores más protegidos de este área.

Este biotopo es característico del Estero Las Montañas (estación 57, Fig. 2k).

Una variante de este biotopo fue el que se observó en Canal Kirke (estación 55), donde sólo Porphyra sp. se encuentra distribuida en el nivel alto, en cambio las restantes especies, Ulva sp., Nothogenia fastigiata, Ceramium sp., Adenocystis utricularis y los balánidos ocupan el nivel medio de la zona eulitoral y la subzona de nivel medio con coberturas claramente menores. El sustrato en general se caracteriza como una pared rocosa vertical levemente inclinada, salinidad de 16 y temperatura de $5,7^{\circ} \mathrm{C}$ (Fig. 2g).

Se conoce una variante poco frecuente observada en el Golfo Elefantes (región de Aysén), donde la zona eulitoral inferior estaba sombreada por rocas sobrepuestas y el conjunto dominado por Heterosiphonia berkeleyii, alga roja confinada normalmente al sublitoral. Comúnmente asociada con el biotopo existe una fauna de moluscos diversa que incluye principalmente las especies Fissurella nigra, Chiton magnificus, Tegula atra, Plaxiphora aurata, Tonicia atra y Nacella magellanica. Si esta alga crece en bolones incluidos en una matriz de arena/gravilla, entonces se asocian con este biotopo especies de poliquetos (e.g., Platynereis australis), cangrejos (e.g., Hemigrapsus crenulatus), anémonas de mar (e.g., Bunodactis sp.) y estrellas de mar (e.g., Comasterias lurida). En playas expuestas a la acción del oleaje Nothogenia podría formar una banda en la zona eulitoral inferior. También se encuentran algas en sectores de playa muy variables que a veces son difíciles de separar en un examen superficial de Iridaea. Es posible que junto con otras algas (e.g., Halopteris, Mazzaella, Nothogenia, Jania) definan sub-biotopos más que biotopos. Las muestras de plantas de Nothogenia más robustas y típicas se han obtenido donde la salinidad es $~ 25$. Esta variante está asociada a condiciones de oleaje protegidas a moderadamente expuestas, comúnmente en la zona eulitoral inferior y con una distribución que va desde Bahía San Quintín (costa Pacífica), Golfo Elefantes, Archipiélago de Los Chonos y lugares con condiciones más marinas dentro de la región de Katalalixar.

\section{Biotopo de IRIDAEA TUBERCULOSA (LR ItUB)}

Zona litoral/rango: Zona eulitoral superior a media.

Clasificación del hábitat: Salinidad 20 hasta totalmente marina ( $\geq 32)$.

Exposición al oleaje: Protegida a moderadamente expuesta.

Otros modificadores: Posiblemente salinidad baja. Ha sido citada para salinidades de 25 .

Distribución geográfica: Se distribuye a través del PNLSR, exceptuando la Laguna San Rafael y el Archipiélago de Los Chonos, en la región de Aysén y en canales interiores de la zona norte y centro de la región de Magallanes.

Descripción: Agregaciones de color café-rojizo o franjas de Iridaea tuberculosa sobre rocas de la subzona eulitoral superior a medio, sobre macizos rocosos y bolones estabilizados, con exposición moderada a protegida frente a la acción del oleaje y que a veces se superponen con el biotopo inferior de Elminius kingii (cirrípedos balánidos). I. tuberculosa suele aparecer mezclada con especies de Porphyra sp. y Ulva sp. en los niveles superiores de zonación y con Pylaiella littoralis y las dos algas anteriores en el nivel medio. En los niveles medios e inferiores es también común encontrar a los moluscos Mytilus chilensis y Aulacomya atra.

En una determinación somera Iridaea tuberculosa puede ser confundida por su morfología externa con Nothogenia fastigiata, la que se distingue por formar diferencia de Nothogenia que es menos membranosacarnosa y de color café rojizo. I. tuberculosa es menos ramificada, y generalmente crece más alta que Nothogenia fastigiata en la zona litoral.

Este biotopo caracteriza a Punta Vergara, Península Antonio Varas (Golfo Almirante Montt) (estación P, Fig. $2 \mathrm{~m})$.

\section{Biotopo de Caepidium antarcticum. (LR CANTa)}

Zona litoral/rango: Zona eulitoral subzona media inferior.

Clasificación del hábitat: Salinidad intermedia (>30) con rango de variación hasta totalmente marina $(\geq 32)$. 
Exposición al oleaje: Moderadamente expuesto a expuesto.

Otros modificadores: Salinidad, tipo de sustrato (rocas), nivel de zonación y competencia por el sustrato (posible pastoreo/herbivoría).

Distribución geográfica: Actualmente restringido a ambientes costeros con influencia oceánica de la zona central de la región de Magallanes.

Descripción: Biotopo representativo de un área semiexpuesta con características marinas (salinidad $\geq 32$ ). Sustrato de paredes inclinadas, macizos rocosos, bloques rocosos y bolones estabilizados, caracterizado por costras y manchones del alga Caepidium antarcticum y algas corallináceas situadas en los niveles medios y bajos; en sectores semi-protegidos se ubica en contacto con Durvillaea antarctica y Macrocystis pyrifera. El biotopo se caracteriza además por la presencia, en forma de parches, de las algas Desmarestia confervoides y Halopteris obovata en niveles medios e inferiores, junto a cirrípedos balánidos (Verruca sp.) y por una importante variedad de especies de moluscos poliplacóforos, bivalvos mitílidos, gasterópodos de los géneros Fissurella y Nacella y actinias, principalmente en niveles inferiores.

Este biotopo no ha sido anteriormente registrado ni descrito para la región de Aysén y corresponde a la Isla Manuel Rodríguez, Canal Smyth (frente a faro Fairway) (estación 61, Figs. 2i y 2n).

\section{Discusión}

Este trabajo constituye la primera propuesta de sintetizar información respecto a los ecosistemas costeros del área comprendida entre Canal Trinidad y Canal Smyth, Patagonia austral, localidades del amplio sector que comenzó a ser investigado por Sköttsberg (1941).

De las 13 estaciones estudiadas durante primavera de 2009, 12 representan una extensión austral de biotopos descritos para sectores más septentrionales de esta región austral de Aysén (John et al. 2002, 2003) (Tabla 2). Sólo el biotopo LR Canta es nuevo y presenta una fuerte influencia subantártica, integrado mayoritariamente por Caepidium antarcticum y algas corallináceas incrustantes distribuidas en la subzona eulitoral media a inferior, en frentes semi-expuestos.

Aunque existen algunas algas que están presentes en más de un biotopo, existe una alta variabilidad en la distribución vertical en los distintos niveles en el sector de zonación intermareal analizado. Del mismo modo es variable su distribución horizontal (cobertura) y la de los invertebrados que conforman cada asociación. Sin embargo, pese a las notables variaciones observadas fue posible individualizar los distintos biotopos que caracterizan las distintas estaciones. En general se observó que existen variaciones latitudinales en las franjas horizontales, representadas por ciertas especies de algas e invertebrados, o grupos de éstos que caracterizan cada uno de los niveles.

Tabla 2. Caracterización de los biotopos intermareales identificados entre Canal Trinidad y Canal Smyth / Characterization of identified intertidal biotopes between Trinidad Channel and Smyth Channel

\begin{tabular}{rrrlll}
\hline Est. & T $\left({ }^{\circ} \mathrm{C}\right)$ & Salinidad & \multicolumn{1}{c}{ Zonación } & Biotopo & Biotopo variante \\
\hline 41 & 8,9 & $30^{*}$ & Eulitoral superior & LR Bhar & \\
75 & 3,7 & $20^{+}$ & Eulitoral superior hasta sublitoral & LR Apac & \\
74 & 5,4 & $22^{+}$ & Eulitotal superior & LR Porp & \\
40 & 7,5 & $31^{*}$ & Eulitoral inferior & LR Autr & \\
43 & 7,3 & $32^{*}$ & Eulitoral superior & LR Porp & LR NothDur \\
60 & 5,8 & $23^{+}$ & Eulitoral superior & LR Bhar & LR AutrNfast \\
55 & 5,7 & $16^{\mathrm{x}}$ & Eulitoral medio e inferior & LR MlamNfast & LR UlAutr \\
$\mathrm{P}$ & 8,5 & $20^{+}$ & Eulitoral superior y medio & LR Itub & \\
$\mathrm{A}$ & 8,9 & $18^{\mathrm{x}}$ & Eulitoral superior & LR BharApac & \\
57 & 6,3 & $25^{+}$ & Eulitoral medio e inferior & LR MlamNfast & \\
68 & 7,4 & $32^{*}$ & Eulitoral superior & LR Bhar & LR Lfau \\
61 & 6,9 & $32^{*}$ & Eulitoral inferior & LR Canta & \\
$\mathrm{F}$ & 6,7 & $17^{\mathrm{x}}$ & Eulitoral superior & LR Porp & \\
\hline
\end{tabular}

*: Condiciones marinas, + : Condiciones de transición de aguas salobres, $\mathrm{x}$ : Condiciones de agua dulce 
Los biotopos estudiados representan asociaciones de organismos que tipifican sectores protegidos y semiexpuestos, o semi-protegidos, debido a la modificación de la influencia del oleaje producida por la protección que proporcionan las islas y accidentes geográficos del sector. Del mismo modo, estos biotopos de frentes semiprotegidos y protegidos presentan una gradual y marcada reducción de la salinidad, a medida que se avanza hacia el sector oriental de la cuenca, producto de la influencia fluvial, junto con la alta pluviosidad y deshielo que caracteriza a los sectores más interiores. Esta situación ha sido confirmada por otros autores (Strub et al. 1998, Dávila et al. 2002) y para organismos planctónicos de la columna de agua (Landaeta et al. 2011).

Del total de estaciones, aquellas situadas más al norte (estaciones 40, 41 y 43) corresponden a los ambientes que presentan características más marinas, con salinidades más altas (30-32) y temperaturas ligeramente más altas $\left(7,3\right.$ a $\left.8,9^{\circ} \mathrm{C}\right)$, seguidas por la estación 68 (32; $7,4^{\circ} \mathrm{C}$ ) y la estación $61\left(32 ; 6,9^{\circ} \mathrm{C}\right)$. En esta última fue donde se distinguió el biotopo nuevo de características más subantárticas (LR Canta). Las estaciones 75, 74, 60, P, y 57 presentaron características intermedias (20 y 25; 3,7 y $8,5^{\circ} \mathrm{C}$ ) y las estaciones A y $\mathrm{F}$ correspondieron a aquellas con mayor influencia de agua dulce $\left(16-18 ; 5,7-8,9^{\circ} \mathrm{C}\right)$.

El nuevo biotopo se extiende desde la zona eulitoral superior hasta la sublitoral. Algunos biotopos de las otras estaciones, caracterizan la zona eulitoral superior (LR Bhar y LR Porp), otros son característicos del eulitoral inferior (LR Canta LR Autr), eulitoral medio e inferior (LR MlamNfast) y eulitoral superior y medio (LR Itub).

El extenso sector austral de Chile constituye, desde Chiloé al Cabo de Hornos, un sistema biogeográfico único (Báez \& Letelier 2001³) que se mantiene como una de las áreas menos conocidas de Chile (Santelices 1991, Silva \& Palma 2006) y del mundo. Esta región, que es considerada una de las áreas estuarinas más extensas del planeta, está siendo transformada aceleradamente. Se hace necesario estudiar la variabilidad estacional de estos biotopos de forma integrada, para fundamentar y documentar los cambios temporales que ocurren en las comunidades intermareales de la región. El conjunto de las especies que integran estos biotopos intermareales constituye, probablemente, una de las reservas de organismos marinos más grande de Chile y forma parte de ecosistemas costeros relativamente vulnerables, si se considera la posibilidad de cualquier cambio en las condiciones físicas del ambiente, como podría ser el calentamiento global, fenómeno que ya empieza a manifestar sus primeros efectos, especialmente sobre los ventisqueros patagónicos (Glasser et al. 2011).

La identificación de las especies de invertebrados bentónicos que caracterizan el biotopo y que constituyen grupos frecuentes y dominantes como moluscos y cirrípedos balánidos (además de las macroalgas), cumplen un rol importante en la ecología de las comunidades biológicas de fiordos y canales del sur de Chile, por lo tanto surge imperativo una completa evaluación y caracterización de la comunidades que habitan los ambientes litorales. Lo anterior no sólo permitirá reconocer asociaciones entre invertebrados y algas, que también constituyen biotopos (véase John et al. 2003), sino que también permitirá realizar comparaciones más precisas respecto a las diferencias biogeográficas que presentan los biotopos latitudinalmente relacionados con diversos factores modificadores, e.g., salinidad, sustrato y tipo de frente.

A menos que una región haya sido cabalmente estudiada, no es posible incluir información sobre variación regional en el hábitat o características de las especies comparadas con aquéllas de otros sitios. Similarmente, sin tener un gran cuerpo de información disponible, es difícil comentar sobre características de interés en la conservación.

En términos generales se ha estimado que, dadas las características de los sitios estudiados donde se efectuaron las recolecciones de material biológico y las observaciones para la determinación y evaluación de los biotopos intermareales, son muy pocos los biotopos que representan ambientes plenamente expuestos, los cuales estarían más bien restringidos a aquellas áreas ubicadas más hacia el oeste, y mucho más cercanas al océano abierto. Mejores condiciones climáticas y oceanográficas en la zona permitirán una mejor evaluación de ambientes más expuestos. Como se ha observado previamente (Báez \& Letelier $2001^{3}$, John et al. 2003), la diversidad de biotopos costeros en esta área es muy variable, condición que es dependiente de la interacción de los múltiples factores abióticos que interactúan en esta extensa región.

Entre estos factores los más importantes son la salinidad, el grado de exposición al oleaje/viento (relacionado con el tipo de frente) y sustrato. En este sentido la salinidad es la variable que denota las mayores diferencias entre un biotopo y otro. Biotopos situados en ambientes con salinidades bajas, con aportes de agua dulce desde el ambiente terrestre (islas, continente) o 
donde la influencia de los hielos producto de la cercanía del ventisquero es acentuada, presentan generalmente riquezas y coberturas menores tanto en macroalgas como en invertebrados marinos. En cambio, biotopos situados en canales y sectores con salinidades mayores, registran habitualmente un mayor número de especies de algas e invertebrados, incluso mayores coberturas y abundancias. Sin embargo, la situación anterior no siempre se explica por una condición oceánica o expuesta del ambiente costero estudiado y podría tener relación con patrones de circulación de las masas de agua que ingresan en los canales interiores (Pickard 1971, Sobarzo 2009). Los complejos patrones de masas de agua y corrientes pueden explicar algunos patrones biogeográficos de los organismos marinos observados (Moreno et al. 2005). Producto de estas condiciones es que la diversidad de organismos marinos, contrariamente a lo que se podría esperar, aumenta hacia las latitudes altas, lo que estaría indicando cuenta de ecosistemas altamente productivos.

En términos generales, la extensa área investigada, unido a la diversidad de hábitats estudiados, permite concluir que no existen grandes diferencias entre los biotopos registrados en el presente trabajo y aquellos señalados anteriormente para el área del Parque Nacional Laguna San Rafael, Estero Elefantes, Archipiélago de Los Chonos (Reserva Nacional Las Guaitecas) y Reserva Nacional Katalalixar, en la región de Aysén.

\section{Agradecimientos}

Los autores desean agradecer a la tripulación del B/O AGOR Vidal Gormaz de la Armada de Chile por el apoyo recibido durante la realización del Crucero CIMAR Fiordos 15 y a una serie de entusiastas científicos que apoyaron la recolección de muestras en terreno. Se agradece también la contribución de los revisores anónimos al presente manuscrito. La presente investigación fue realizada en el marco del Proyecto 'Biotopos Marinos Intermareales y de Aguas Someras entre Canal Trinidad y Canal Smyth, XII Región’ (C15F 09-13) financiada por el Comité Oceanográfico Nacional.

\section{LITERATURA CITADA}

Antezana T. 1999. Hydrographic features of Magellan and Fuegian inland passages and adjacent Subantarctic waters. Scientia Marina 63 (Suppl. 1): 23-34.

Araya J. 1999. Perfiles longitudinales de fiordos de Patagonia Central. Ciencia y Tecnología del Mar 22: 3-30.

Arntz W \& C Ríos. 1999. Magellan-Antarctic: Ecosystems that drifted apart. Scientia Marina 63 (Suppl. 1): 503-511.
Arntz W, G Lovrich \& S Thatje. 2005. The Magellan-Antarctic connection: Links and frontiers at high southern latitudes. Scientia Marina 69 (Suppl. 2): 359-365.

Buschmann A, V Riquelme, M Hernández-González, D Varela, J Jiménez, L Henríquez, P Vergara, R Guiñez \& L Filún. 2006. A review of the impacts of salmonid farming on marine coastal ecosystems in the southeast Pacific. ICES Journal of Marine Science 63: 1338-1345.

Bustos CA, MF Landaeta \& F Balbontín. 2008. Efectos ambientales sobre la variabilidad espacial del ictioplancton de Chile austral durante noviembre de 2005. Revista Chilena de Historia Natural 81: 205-219.

Camus P. 2001. Biogeografía marina de Chile continental. Revista Chilena de Historia Natural 74: 587-617.

Connors D, D Brazier, T Hill \& K Northern. 1997. Marine biotope classification for Britain and Ireland. V.1 Littoral biotopes. Joint Nature Conservation Committee Report 229: 1-362.

Dávila P, D Figueroa \& E Müller. 2002. Freshwater input into the coastal ocean and its relation with the salinity

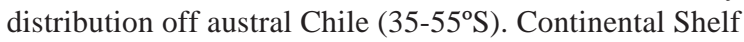
Research 22: 521-534.

Försterra G. 2009. Ecological and biogeographical aspects of the Chilean Fjord Region.In: Häussermann V \& G Försterra (eds). Marine benthic fauna of Chilean Patagonia, pp. 6176. Nature in Focus, Santiago.

Guzmán L \& C Ríos. 1986. Análisis de la estructura en comunidades intermareales archipiélago de Cabo de Hornos: ambientes de bloques y cantos. Estudios Oceanológicos 5: 67-105

Häussermann V \& G Försterra. 2009. Marine benthic fauna of Chilean Patagonia, 1000 pp. Nature in Focus, Santiago.

Hervé F, D Quiroz \& P Duhart. 2009. Principal geological aspects of the Chilean Fjord Region. In: Häussermann V \& G Försterra (eds). Marine benthic fauna of Chilean Patagonia, pp. 30-42. Nature in Focus, Santiago.

Glasser N, S Harrison, K Jansson, KAnderson \&A Cowley. 2011. Global sea-level contribution from the Patagonian Icefields since the Little Ice Age maximum. Nature Geoscience 4: 303-307.

Hinojosa I, M Pizarro, M Ramos \& M Thiel. 2010. Spatial and temporal distribution of floating kelp in the channels and fjord of southern Chile. Estuarine, Coastal Shelf Science 87: 367-377.

John D, G Paterson, N Evans, M Ramirez, M SpencerJones \& D Reid. 1998. A provisional manual of marine biotopes of the Laguna San Rafael National Park and Estero Elefantes, southern Chile, 37 pp. The Natural History Museum, London [CD-ROM version]

John D, R Foster-Smith, G Paterson, M Ramírez, N Evans, M Spencer-Jones, D Reid \& T Ferrero. 2002. A 'biotope' approach to the marine benthic biological assemblages of the Laguna San Rafael National Park, Chile. Boletín del Museo Nacional de Historia Natural, Chile 51: 159-173. 
John D, G Paterson, N Evans, M Ramirez, M SpencerJones, P Baez, T Ferrero, C Valentine \& D Reid. 2003. Manual de biotopos marinos de la Región de Aysén, Sur de Chile. : Parque Nacional Laguna San Rafael, Estero Elefantes, Archipiélago de los Chonos y Katalalixar, 127 pp. Biodiversity Aysén Project, Department of Zoology, Natural History Museum, London.

Landaeta MF, CA Bustos, P Palacios-Fuentes, P Rojas \& F Balbontín. 2011. Distribución del ictioplancton en la Patagonia austral de Chile: potenciales efectos del deshielo de Campos de Hielo Sur. Latin American Journal of Aquatic Research 39(2): 236-249.

Lewis J. 1964. The ecology of rocky shores, 323 pp. The English University Press, London.

Marín V \& L Delgado. 2009. Diversidad y distribución espacial de copépodos superficiales (0-10 metros) en la zona costera del mar interior de Chiloé (CIMAR 10 Fiordos). Ciencia y Tecnología del Mar 32(1): 95-100.

Moreno R, C Hernández, M Rivadeneira, M Vidal \& N Rozbaczylo. 2005. Patterns of endemism in southeastern Pacific benthic polychaetes of the Chilean coast. Journal of Biogeography 33(4): 750-759.

Olenin S \& JP Ducrotoy. 2006. The concept of biotope in marine ecology and coastal Management. Marine Pollution Bulletin 53: 20-29.

Osorio C, M Ramírez \& M Vega. 2002. Distribución y abundancia de macroorganismos del intermareal de Isla Traiguén $\left(45^{\circ} \mathrm{S}, 73^{\circ} \mathrm{W}\right)$ Estero Elefantes, Región de Aisén, Chile. Boletín del Museo Nacional de Historia Natural, Chile 51: 175-184.

Palma S \& N Silva. 2004. Distribution of siphonophores, chaetognaths, euphausiids and oceanographic conditions in the fjords and channels of southern. Deep-Sea Research 51(2): 513-535.

Pickard G. 1971. Some physical oceanographic features of inlets of Chile. Journal of the Fisheries Research Board of Canada 28: 1077-1106.

Ramajo L \& C Osorio. 2010. Riqueza, abundancia y patrones espaciales de moluscos desde el estero Reloncaví (41³3’S) a la Boca del Guafo (43 49'S), sur de Chile. Ciencia y Tecnología del Mar 33(1): 57-65.

Ramírez M. 2010. Flora marina bentónica de la Región Austral de Sudamérica y la Antártica. Anales del Instituto de la Patagonia, Chile 38(1): 34-55.

Reid D \& C Osorio. 2000. The shallow-water marine Mollusca of the Estero Elefantes and Laguna San Rafael, southern Chile. Bulletin of the Natural History Museum, Zoology Series 66(2): 109-146.
Ríos C \& L Guzmán. 1982. Reevaluación de la estructura de la comunidad en una playa de bloques y cantos de alta latitud (Archipiélago de Cabo de Hornos). Anales del Instituto de la Patagonia Series Ciencias Naturales 13: 211-224

Santelices B. 1991. Littoral and sublittoral communities of continental Chile. In: Mathieson AC \& PH Niehuis (eds). Intertidal and littoral ecosystems. Ecosystems of the World 24: 347-369. Elsevier, London.

Scrhödl M. 2003. Sea slugs of Southern South America, 165 pp. ConchBooks. Hackenheim.

Silva N \& S Palma. 2006. Avances en el conocimiento oceanográfico de las aguas interiores chilenas, Puerto Montt a Cabo de Hornos, 162 pp. Comité Oceanográfico Nacional, Pontificia Universidad Católica de Valparaíso, Valparaíso.

Silva N, C Calvete \& H Sievers. 1997. Características oceanográficas y químicas de canales australes chilenos entre Puerto Montt y Laguna San Rafael. (Crucero Cimar-Fiordo I). Ciencia y Tecnología del Mar 20: 23-106.

Sköttsberg C. 1941. Communities of marine algae in subantarctic and antarctic waters. Kongliga Svenska Vetenskaps Academiens Handlingar, Series 3, 19(4): 1-92.

Sobarzo M. 2009. The southern Chilean fjord Region: Oceanographic aspects. In: Häussermann V \& G Försterra (eds). Marine benthic fauna of Chilean Patagonia, pp. 5360. Nature in Focus, Santiago.

Soto E \& G Paterson. 2010. Poliquetos bentónicos intermareales y sublitorales de la región de Aisén, Chile. Anales del Instituto de la Patagonia, Chile 38(2): 69-80.

Soto M. 2009. Geography of the Chilean Fjord Region. In: Häussermann V \& G Försterra (eds). Marine benthic fauna of Chilean Patagonia, pp. 43-52. Nature in Focus, Santiago.

Stephenson T \& A Stephenson. 1949. The universal features of zonation between tide marks on rocky coasts. Journal of Ecology 37: 289-305.

Stephenson T \& A Stephenson. 1972. Life between tidemarks on rocky shores, 425 pp. W. H. Freeman, San Francisco.

Strub P, J Mesías, V Montecino, J Rutllant \& S Salinas. 1998. Coastal ocean circulation off western South America. In: Robinson AR \& KH Brink (eds). The Sea 11: 273-313. John Wiley \& Sons, New York.

Thatje S \& A Brown. 2009. The macrobenthic ecology of the Straits of Magellan and the Beagle Channel. Anales del Instituto de la Patagonia, Chile 37(2): 27-37.

Recibido el 22 de septiembre de 2011 y aceptado el 10 de mayo de 2012

Editor Asociado: Mauricio Landaeta D. 